\title{
Cólico nefrítico y hallazgo casual torácico
}

\author{
Martín García AMª ${ }^{1}$, Hernández Navarro JC. ${ }^{2}$, Baquero López FR. ${ }^{3}$
}

Sanid. mil. 2011; 67 (3): 323-324; ISSN: 1887-8571

Varón de 36 a que padece cólico renoureteral litiásico izquierdo y se realiza RX de abdomen y tórax (Figuras 1 y 2) en urgencias. Tras la resolución del cuadro urológico, es remitido a Radiología por el neumólogo para realizar ecografía torácica para estudio de posible derrame pleural y marcaje en piel para toracocentesis.
La ecografía descarta derrame, tampoco encapsulado subpulmonar. En la parte inferior del hemitórax derecho existe un tejido ecogénico-sólido de naturaleza indeterminada. Se realiza TAC (Figuras 3-6).

El único antecedente referido era una caída de moto hace varios años.

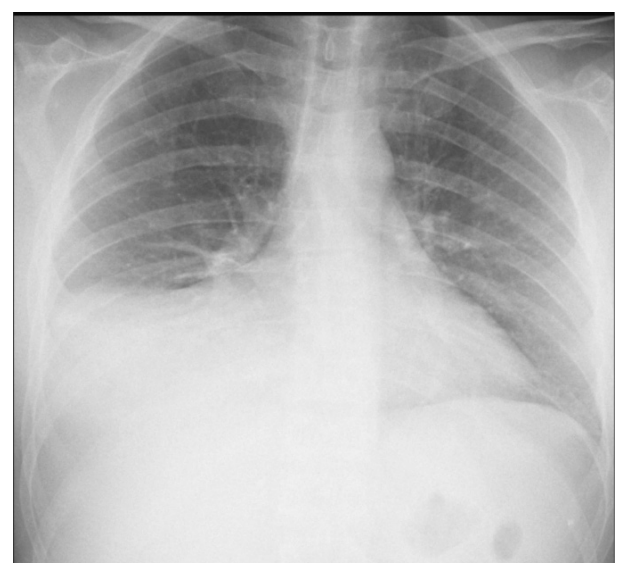

Figura 1

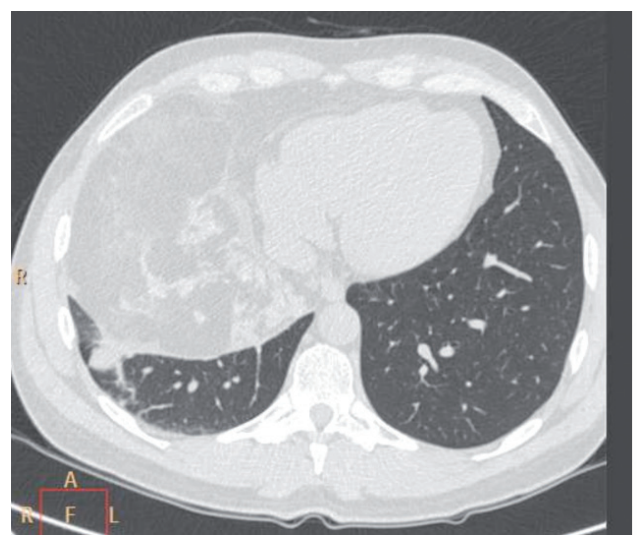

Figura 4

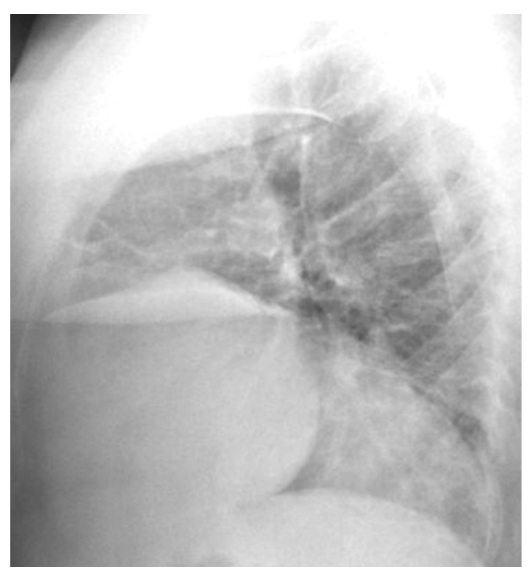

Figura 2

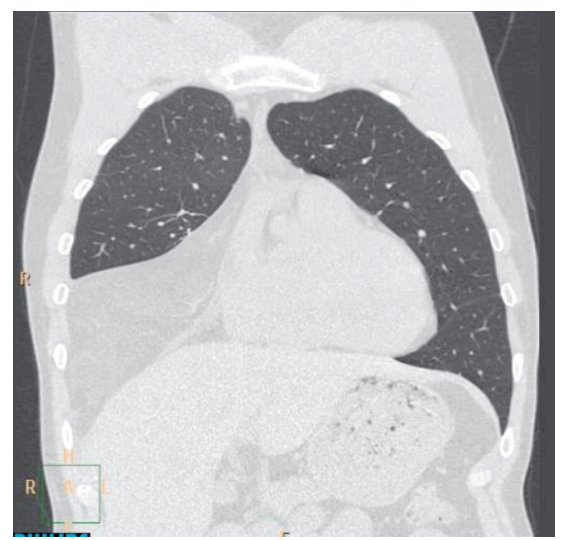

Figura 5

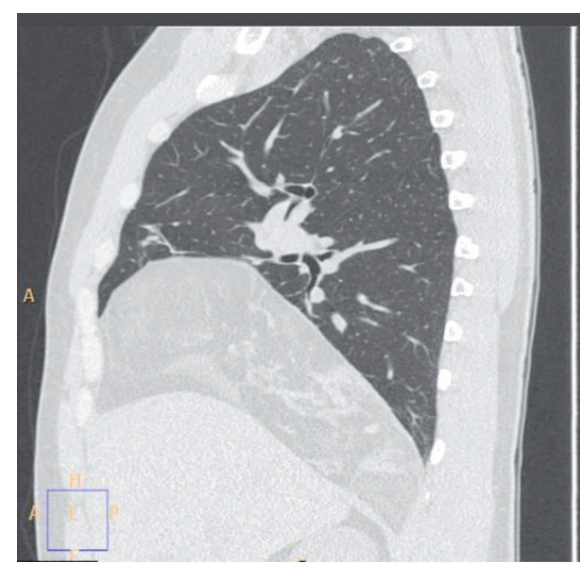

Figura 3

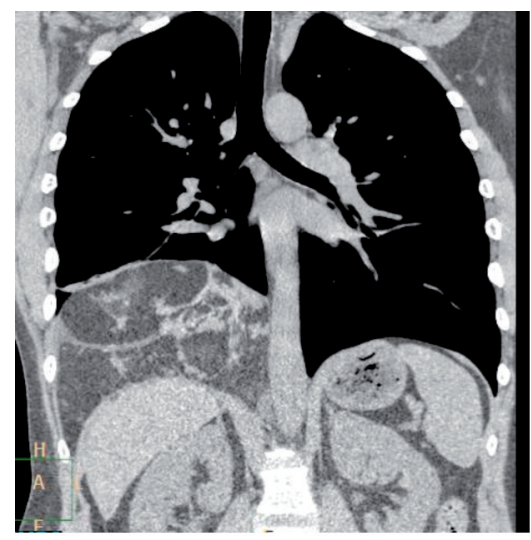

Figura 6

${ }^{1}$ Cte. Médico. Servicio de Radiodiagnóstico.

${ }^{2}$ Col Médico. Servicio de Radiodiagnóstico.

${ }^{3}$ Tcol Médico. Servicio de Cirugía Torácica.

Hospital Central de la Defensa Gómez Ulla.

Dirección para correspondencia: A. María Martín García. Servicio de Radiodiagnóstico.

Hospital Central de la Defensa Gómez Ulla. Glorieta del Ejército s/n. 28047 Madrid.

Recibido: 3 de marzo de 2010

Aceptado: 26 de mayo de 2011 


\section{Diagnóstico: Timolipoma}

Tras la realización de la ecografía y la consulta al clínico, se procede a realizar TC que objetiva un material de atenuación dominante en rango de grasa con tractos o cordones de mayor atenuación. En su conjunto se adapta a estructuras anatómicas adyacentes y la localización dominante es subpulmo- nar derecha. No existen discontinuidades frénicas, ni lesiones costales.

Existe una fina conexión aparente anterior y superior con el mediastino.

Fue extirpada una masa de consistencia blanda de 1.365 gr .

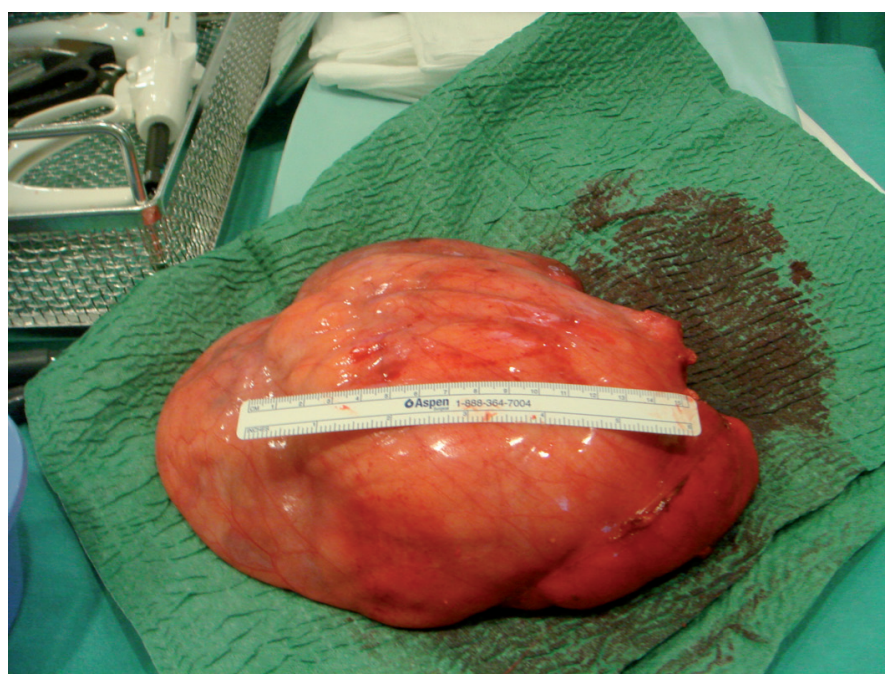

Figura 7

El timolipoma es una tumoración benigna rara que puede crecer mucho sin dar síntomas, debido a que no infiltra y se adapta a estructuras adyacentes con localizaciones intratorácicas que pueden resultar «atípicas» a pesar de su dependencia mediastínica.

Diagnóstico diferencial: debido al componente dominante graso y a la situación inferior en el tórax así como a los «cordones»y reticulación interna, con el antecedente antiguo traumático, nos hizo pensar en primer lugar en la posibilidad de herniación diferida por rotura frénica. Se descartó algún tipo de continuidad trasfrénica.

La conexión mediante prolongación estrecha demostrada en los cortes coronales anteriores (Figura 5 flechas) con el mediastino anterosuperior dio la clave para sugerir el diagnóstico que se confirmó con la cirugía y anatomía patológica.
En la bibliografía se habla de timolipomas gigantes ${ }^{1-3}$ pero no hemos encontrado referencias de tamaños mayores que el que mostramos.

\section{BIBLIOGRAFÍA}

1. C. Roque, P. Rodríguez, C. Quintero, N. Santana, M. Hussein and J. Freixineta; Timolipoma gigante; Archivos de Bronconeumología Volume 41, Issue 7, July 2005, Pages 402-403.

2. Naidich,Webb, Müller, Krinsky, Zerhouni, Siegelman -»Tórax, TC y RM». Pag. 74.Edit Marban $3^{\mathrm{a}}$ Ed 2000.

3. W. Richard Webb, Charles B. Higgins»Thoracic Imaging»Pag.223-225 Edit Lippincott Williams\& Wilkins. 2005. 